\title{
LÍNEAS DE INVESTIGACIÓN Y TENDENCIAS DE LA EDUCACIÓN A DisTANCIA EN AMÉRICA LATINA A TRAVÉS DE LAS TESIS DOCTORALES
}

\author{
(RESEARCH AND TRENDS OF DISTANCE EDUCATION IN LATIN AMERICAN THROUGH THE \\ DOCTORALTHESIS)
}

\author{
María García Pérez \\ Lorenzo García Aretio \\ Universidad Nacional de Educación a Distancia, UNED (España)
}

\section{RESUMEN}

El propósito de este artículo es examinar, comprender y analizar las características y tendencias generales de las tesis doctorales defendidas en torno a la educación a distancia en América Latina. Para este fin, esta investigación se ha compuesto de dos fases, la primera realiza un análisis descriptivo de un total de 183 documentos defendidos entre los años 2004 y 2011. En esta parte se analizan las tendencias y necesidades de la educación a distancia cómo áreas de investigación analizadas en las tesis doctorales. Y en la segunda fase, a través de análisis de referencias bibliográficas se detallan las referencias de citación utilizadas como indicador de consumo. Los resultados obtenidos muestran que la dispersión de temas de investigación no ha variado significativamente y que existen dos áreas dentro de la educación a distancia que son propuestas para futuras investigaciones.

Palabras clave: América latina, investigación en educación a distancia, áreas de investigación, bibliometría.

\begin{abstract}
The purpose of this article is to examine, understand and analyze the characteristics and general trends of doctoral theses about distance education in Latin America. To this end, this research is composed of two phases, the first performed a descriptive analysis of a total of 183 documents defended between 2004 and 2011. In this part the trends and needs of distance education how research areas discussed in the doctoral thesis analyzed. In the second phase, through analysis of references citing references used as an indicator of consumption are detailed. The results show that the dispersion of research topics has not changed significantly and that there are two areas within the distance education are proposed for future research.
\end{abstract}




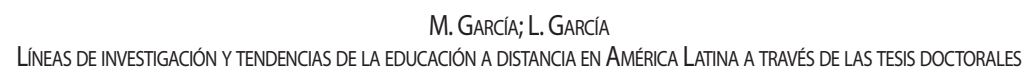

Keywords: Latin American, review of distance education research, research areas, bibliometric.

La importancia que la ciencia ha llegado a adquirir en nuestra sociedad se fue imponiendo a lo largo de los años y se consolidó durante todo el siglo XX. Este desarrollo pronto necesitó de la evaluación de las actividades y los recursos que los investigadores dedicaban a la propia ciencia así como del medio en que ésta era difundida. Surgieron así variadas formas para abordar tanto los distintos aspectos de la actividad científica como la propia evolución de la ciencia.

Entre los variados estudios en torno a la propia ciencia hay una cuestión que ha requerido la atención de muchos investigadores: el análisis de los resultados de la producción científica o conocimiento científico. Desde este prisma, nos cuestionamos sobre qué pretenden la sociedad y los investigadores con la medición de la producción científica y sobre los medios y las fuentes existentes para llevar a cabo esta labor. Estas y otras preguntas son a las que se pretende dar respuesta a lo largo de este artículo. Preguntas y respuestas que deseamos centrar en un campo concreto de análisis y estudio, el de la educación a distancia.

El objeto de la labor investigadora es siempre la publicación y difusión de los resultados fruto de la misma. El momento en el que se comunican públicamente esos resultados nos permite encontrar un espacio para compartir y debatir sobre el conocimiento generado, con el resto de la comunidad científica, lo que a su vez permite asegurar su replicabilidad para construir entre todos los investigadores nuevos conocimientos, que es principio fundamental de toda investigación que pretenda catalogarse como científica.

Esta difusión y comunicación de resultados por parte del investigador a toda la comunidad científica, se ha realizado tradicionalmente por diferentes vías, tales como los libros y los capítulos de libros, las tesis doctorales, informes de investigación, artículos en revistas científicas, comunicaciones y ponencias presentadas en congresos y seminarios, etc.

Así, la divulgación del conocimiento científico contribuye al desarrollo de la sociedad, ya que como señalan Solano y otros (2009: 59) "la investigación científica y tecnológica y la difusión de los conocimientos constituyen actividades esenciales para la satisfacción de las crecientes necesidades sociales".

La ciencia con mayor impacto es aquella cuyos frutos se transmiten mejor, por lo tanto, comunicar los resultados de ésta es tan importante como la ciencia 
misma (Clark, 2005). La productividad científica se refleja en las comunicaciones formales vía publicaciones avaladas por el mismo sistema científico. Este sistema está constituido por la instituciones, asociaciones y colegios de científicos que, con el fin de evaluar la calidad y relevancia de los productos, han generado un conjunto de normas y reglas de lo que se espera para ser un buen científico, con una posición reconocida en la estructura organizacional de la ciencia y con acceso igual a los recursos y oportunidades de reconocimiento y financiamiento.

\section{LA ACTIVIDAD CIENTÍFICO-INVESTIGADORA Y SU TRANSMISIÓN}

Para Ziman (1972) la ciencia debe ser entendida como un conocimiento público, y desde este prisma destaca el carácter social y acumulativo del conocimiento dentro de la sociedad. Para el autor, este tipo de conocimiento sólo es posible gracias a la comunicación que los investigadores hacen de sus trabajos y que aportan elementos para el desarrollo de la ciencia. Desde este mismo enfoque, Mialtras (1996) sostiene que el conjunto de las aportaciones que se realizan mediante la comunicación no debe convertirse en una mera acumulación de datos o de conclusiones, sino que lo fundamental es su transmisión para que sirvan como fuente de inspiración para los otros investigadores.

En los países de América Latina, según cada uno de los casos, existen muchas limitaciones y dificultades provenientes de la necesidad de reducir los problemas sociales e impulsar el crecimiento económico (Quintanilla-Montolla, 2010). No obstante, en las últimas décadas estos países han dado un salto considerable, tanto cuantitativo como cualitativo, en lo que se refiere a producción científica (SaavedraFernández y otros, 2002). Así se han asentado unas bases sólidas y se han buscado nuevos objetivos más ambiciosos, considerando la producción científica como un poderoso instrumento de transformación social y desarrollo económico.

La ciencia en todos los tiempos y lugares se ha sustentado en la comunicación como elemento sustancial, ya que sólo existe si se respalda en el conocimiento público. Ya Ziman (1980) defendía que la ciencia dependía de la comunicación por varias razones:

- Es vital crear un registro permanente de resultados, observaciones y teorías que sirvan de referencia para el resto de la comunidad científica.

- Es esencial refutar, perfeccionar y criticar los resultados de las investigaciones realizadas en pro de la ciencia en sí misma. 
Partiendo de esta base que nos ofrece el autor, resulta necesario plantearse el propio objetivo de una investigación y reflexionar sobre la importancia de que una dimensión de este propio elemento es su difusión, consumo y diseminación por todos los investigadores. Por tanto, resulta evidente que la transmisión, comunicación de resultados de un trabajo científico, es imprescindible en cualquiera de sus cauces establecidos para este fin. O como señala Price (1978) "el acto de la creación de la investigación científica está incompleto sin la publicación, puesto que es la publicación la que proporciona el proceso conectivo, la evaluación, y quizás, el asentimiento de la comunidad científica”. Así la comunicación juega un papel clave en el mantenimiento, organización y futuro de la propia ciencia en su totalidad, ya que permite la adquisición del conocimiento de la situación de los problemas planteados y el resultado de las acciones realizadas para subsanar los mismos.

No hay que olvidar que en el objetivo marcado dentro de las universidades lo que se persigue es la producción de conocimiento científico. Como señalaban López Piñero y Terrada (1992: 66) "este objetivo se cumple solo cuando, independientemente de cuál sea la dimensión de la investigación científico-técnica, el investigador pone a disposición de la comunidad científica su aportación para que puedan beneficiarse de dichas contribuciones los demás investigadores”. Y Ziman (1980) profundizando aún más sobre lo dicho, puntualiza que sólo de esta manera las teorías y los resultados de las contribuciones pueden ser criticados, refutados o mejorados.

El intercambio científico trae como consecuencia la plena realización de la ciencia, una dimensión práctica más allá de la propia acción científica. La ciencia necesita de la comunicación para asegurar su evolución y rigor, por lo que la importancia y abundancia de los cauces de transmisión de esas investigaciones aumentan las posibilidades de desarrollo y potencian que su dispersión sea mayor.

La producción académica y científica se origina bien como consecuencia asociada con la investigación y la docencia, o bien por las actividades académico-universitarias de carácter transversal que de igual manera fundamentan los saberes científicos a través de la práctica. O dicho de otro modo, según la ACAP (2007: 24) "la función investigadora consiste en la realización de actividades intelectuales y experimentales de modo sistemático con el propósito de aumentar los conocimientos sobre una determinada materia. En la medida en que se trata de ampliar el conocimiento, la actividad investigadora puede consistir en la creación de dicho conocimiento, en la realización de estudios profundos o en una labor de síntesis del conocimiento ya generado por otros". 
En la mayoría de los casos la comunicación y transmisión de la información entre los investigadores se ha producido a través de la producción científica escrita. Para Price (1963) la investigación escrita "no es un subproducto del trabajo que se está realizando, sino el producto final de la actividad científica”. Sin embargo, como se señalaba anteriormente, existen diversas formas de comunicación para transmitir el conocimiento científico. Fernández Quijada (2008) afirma que en el ámbito de las ciencias sociales, donde estaría enmarcada la educación a distancia, los libros son considerados la forma habitual de difusión de su investigación científica. Es lo que este autor ha denominado la "cultura de la monografía" (2008: 6) y que establece al libro en una posición preeminente como medio de difusión del trabajo científico frente a otro tipo de publicaciones.

\section{TESIS DOCTORALES SOBRE LA EDUCACIÓN A DISTANCIA}

Aunque el análisis de la investigación no es algo nuevo, su necesidad es más acuciante en la actualidad debido a la enorme cantidad de producción científica que surge cada año especialmente en un producto tan importante como son las tesis doctorales.

La investigación es un componente vital para el desarrollo académico profesional de los futuros doctores. En este contexto, el trabajo presentado como tesis debe ser un ejercicio donde se demuestre el dominio de destrezas para la investigación y la capacidad de trabajo heurístico en un determinado campo de estudio, en este caso la educación a distancia. Es decir, el doctorando debe ser capaz de demostrar la pertinencia científica y profesional del tema elegido, su conocimiento y la disponibilidad de fuentes y referencias bibliográficas significativas, así como el manejo de técnicas de información válidas y estrategias de investigación propias de la disciplina.

Por tanto, como afirman Repiso, Torres y Delgado (2013), las tesis doctorales, desde la perspectiva de la investigación, son documentos que se caracterizan por ser una investigación original en un campo determinado, además hay que destacar la exhaustiva revisión bibliográfica que suele acompañar a este tipo de trabajos (Miguel, 2000). Cuando hablamos del campo de la educación a distancia, es importante resaltar que el mismo se ha ampliado como resultado de las propias investigaciones y trabajos evaluativos realizados sobre esta modalidad.

Aparte de representar la culminación del ciclo formativo de un universitario, la tesis doctoral es el primer trabajo científico y el más importante (Repiso, Torres y Delgado, 2013). Pero, ante todo es un trabajo de investigación que debe fundamentar 
su importancia y la necesidad de esta investigación, esto es, debe aportar nuevo conocimiento. Y para acreditarlo se establece un sistema de control y de evaluación mediante un tribunal de expertos en el área que lo certifica con unos criterios más exigentes que los propios artículos publicados en las revistas científicas. Como destaca Muñoz (2005), es fundamental otorgar a las tesis doctorales la importancia científica que tienen al proporcionar una imagen fiel de las nuevas vías de investigación. Por consiguiente, las tesis doctorales son los espejos en que se reflejan las líneas y tendencias de gran parte de la comunidad científica. En definitiva, son un excelente referente para conocer la estructura, líneas y tendencias de la investigación, en nuestro caso, en el campo de la educación a distancia en el ámbito de América Latina.

Según López López (1996), una de las fuentes documentales más importantes para estudiar el estado de la investigación de un país, a través de su literatura científica, es la producción correspondiente a las tesis doctorales. Uno de los aspectos más relevantes es que este tipo de investigaciones están sometidas al escrutinio y examen de un tribunal, lo que parece obligado en todo caso que reúna del modo más completo posible los requisitos formales exigidos por la investigación científica (Sierra Bravo, 1996). Buela-Casal (2005), al igual que Moyano y otros (2006) resaltan su importancia como medida para comprobar el potencial de formación de nuevos investigadores. Así, pueden constituir un elemento esencial para medir la investigación, en este caso, en el campo de la educación a distancia.

Así, en este trabajo utilizaremos como fuentes documentales las tesis doctorales. Es sabido que las fuentes primarias de donde se nutren muchos artículos de investigación han sido precisamente las tesis (Sierra Bravo, 1996), por lo que se comienzan a estudiar dentro de la literatura científica como objetos propios de investigación en algunos países, aunque todavía no ha sido el caso del área latinoamericana.

Lo dicho hasta aquí evidencia la importancia de la tesis doctoral como trabajo de investigación y, por tanto, su análisis se presenta como un medio idóneo para estudiar la evolución de la investigación científica en cualquier área del saber, y en el caso que nos ocupa, en la educación a distancia en América Latina.

\section{SITUACIÓN ACTUAL. REVISIÓN DE LA LITERATURA}

La educación a distancia ha jugado y tiene en la actualidad un papel relevante en el desarrollo de la educación. Por esta razón, este campo ha crecido y evolucionado, influenciado principalmente por los recursos que ha ofrecido Internet para la mejora de su metodología (García Aretio, 2014). 
Existen numerosos estudios en torno a la educación a distancia, y más aún en la aplicación de la tecnología a la educación, aunque como afirma Moore (2000) es necesario profundizar en aquello que ya se ha investigado en esta modalidad. Si hacemos una revisión de la literatura podremos comprobar cómo muchos investigadores han analizado la evolución y tendencias de la educación a distancia mediante el análisis de revistas nacionales e internacionales (Hew, Kale, y Kim, 2007; Hranstinski y Keller, 2007; Latchem, 2006; Ma, 2000; Ross, Morrison, y Lowther, 2010). A continuación se presentan algunas investigaciones realizadas en esta área a modo de ejemplos para constatar la relevancia y fundamentos de la revisión de la literatura.

Un ejemplo de estas investigaciones fue realizada a nivel internacional por Bonk y Dennen (2003) que identificaron puntos de referencia y las mejores prácticas en torno a la planificación de este método educativo. Estos investigadores realizaron un estudio comparativo de análisis de calidad de la educación a distancia en las universidades a través de los documentos ofrecidos por éstas.

El objetivo principal de Moore y Anderson (2003) en su libro "Handbook of distance education" fue proporcionar una recopilación exhaustiva de la literatura existente como una referencia para los profesionales de la tecnología educativa en respuesta a los cambios y avances en la educación a distancia. Otros investigadores como Anglin y Morrison, 2000; Berge y Mrozowski, 2001; Koble y Bunker, 1997; Lee, Driscoll, y Nelson, 2004; Rourke y Szabo, 2002 han analizado y sintetizado igualmente las publicaciones a través de una revisión con el mismo propósito. Gibson (2003) utilizó los indicadores bibliométricos revisando los artículos publicados en revistas de tres países (Estados Unidos, Canadá y Australia), pero dirigida específicamente a la investigación sobre el aprendizaje y los estudiantes en la educación a distancia.

Moore y Kearsley (2005) analizaron los temas de investigación publicados entre los años 2002 a 2006 y el sistema de evaluación en las cuatro revistas que para ellos eran las más relevantes en el campo de la educación a distancia que son: American Journal of Distance Education, The Journal of Distance Education (JOFDE), Open Learning, y Distance Education (de Australia).

No obstante, otros autores como Berge y Mrozowski (2001) utilizaron también estas mismas revistas para analizar el sistema de evaluación de artículos y la calidad de las contribuciones científicas en esta área de investigación. 
Otros investigadores han analizadola evolución de la educación a distancia a través de la revisión y los indicadores bibliométricos de una sola publicación especializada en este campo específico. Tal es el caso de Koble y Bunker (1997) que estudiaron las tendencias de investigación en el campo de la educación a distancia a través de la revista American Journal of Distance Education en el periodo comprendido entre 1987-1995, pero existen otros casos como es el de Rourke y Szabo (2002) quienes analizaron la revista Journal Distance Education (JDE) desde 1986 hasta 2001. Y Perraton (2000) quien investigó los trabajos de investigación publicados en torno a la educación a distancia especialmente en la revista IRRODL clasificando los temas de la investigación en cuatro áreas específicas.

En Estados Unidos, organismos como el Institute for Higher Education Policy (IHEP), también han analizado las publicaciones en torno a esta misma cuestión. El primero de los informes que publicó el IHEP fue en 1999 y su objetivo se centró en la eficacia y deficiencias de la investigación en la educación a distancia en las universidades a través de diversos documentos. Posteriormente, el IHEP publicó un segundo informe en el año 2000 y en dicho documento identificó 45 referencias agrupadas en siete categorías llamadas los puntos de referencia para la mejora de la calidad de la educación superior a distancia. El objetivo principal de este segundo informe fue ofrecer una base para futuras investigaciones y análisis en torno a la educación a distancia.

Vista la relevancia de las revisiones de literatura científica para el desarrollo del conocimiento, en nuestro caso, como ya adelantábamos, nos centraremos en las fuentes primarias que suponen las tesis doctorales.

\section{MATERIALES Y MÉTODOS}

Realizamos un estudio descriptivo longitudinal retrospectivo de las tesis doctorales sobre educación a distancia de doctorandos de América Latina entre los años 2004 y 2011.

Para localizar las tesis doctorales se han utilizado varias fuentes de información debido a la dificultad de localización de las mismas, ya que no se trata de tesis que hayan sido defendidas en estas regiones en concreto, sino de tesis que han sido defendidas por doctorandos latinoamericanos. Por ello las bases de datos utilizadas han sido:

- Biblioteca Virtual Miguel Cervantes: Tesis doctorales defendidas en cualquier país del mundo en lengua hispana. 
- REDIAL: es la Red Europea de Información y Documentación sobre América Latina donde se recogen tesis que traten sobre temas de América Latina.

- Networked Digital Library of Theses and Dissertations (NDTLD): es una organización internacional dedicada a promover la adopción, creación, uso, diseminación y preservación de tesis y disertaciones electrónicas (ETDs).

- Tesis y monografías de graduación de la Facultad de Ciencias Económicas y Sociales de la Universidad Nacional de Mar del Plata.

- Biblioteca Digital Brasileira de Teses e Dissertaçoes (BDTD).

- Biblioteca Digital de Teses e Dissertações da Universidade de São Paulo (USP).

- TESEO.

- EThOS (Electronic Theses Online Service) es una base de datos de tesis de la British Library.

- Cybertesis: auspiciada por la UNESCO y contando con varios países de América Latina, entre los que destacan Bolivia, Perú y Chile donde se han unido y clasificado las tesis doctorales defendidas en varias universidades de esta región.

- $\quad$ Dialnet Tesis.

- TDR (Tesis Doctorales en Red) es un repositorio cooperativo que contiene, en formato digital, las tesis doctorales leídas en las universidades españolas.

- MIT Theses es una colección de tesis doctorales seleccionadas y que han sido defendidas en EEUU.

- NCSU Theses and Dissertations de North Carolina State University.

- TESIUAMI: repositorio de Tesis de la Universidad Autónoma Metropolitana de México.

- Tesis digitales a texto completo de la Universidad de las Américas de México.

- $\quad$ RPTD (Red Peruana de Tesis Digitales): es una iniciativa de la Universidad Nacional Mayor de San Marcos (UNMSM). 
Para recuperar los documentos pertinentes se aplicó la siguiente estrategia de búsqueda: "Educación a distancia” OR "E-learning” OR "Educación en línea" OR Educación virtual" OR "Educación online" OR "Distance education" OR "Distance Learning” OR "ITC” $O R$ “TIC”. La búsqueda se realizó desde el 17 de diciembre de 2012 al 3 de enero de 2013. Este periodo se alargó en el tiempo por la gran cantidad de bases de datos de tesis consultadas y la abundancia de documentos que fueron eliminados por no ser pertinentes o no poder acceder a ellos en línea. Una vez que se obtuvieron los documentos se creó una base de datos propia y relacional en Microsoft $®$ Office Excell para su posterior tratamiento.

Al introducir los datos en la base de datos propia se crearon una serie de apartados que son necesarios para llegar a los resultados de esta investigación. Las variables que se han introducido para la recopilación son:

- Nombre del autor.

- País de procedencia del autor.

- Nombre del director de la tesis.

- Universidad donde se ha defendido la tesis.

- Año de defensa.

- Título de la tesis.

- Link de la tesis.

- Categoría y subcategoría según los criterios del análisis de contenidos.

Aunque no se puede afirmar que las 183 tesis identificadas en este estudio coincidan con exactitud con las realmente leídas en el campo de la educación a distancia por doctorandos de América Latina entre los años 2004- 2011, sí se puede señalar que los documentos analizados son representativos y pueden mostrar las tendencias en esta metodología educativa. No obstante, es importante tener en cuenta este elemento ya que este hecho podría producir pequeños sesgos en el conjunto de todos los datos.

La relevancia de este análisis radica en que no existen hasta el momento estudios bibliométricos sobre la educación a distancia a través de tesis doctorales defendidas por doctorandos latinoamericanos. Si bien, como se decía en un apartado anterior, 
sí que existen publicaciones sobre el área de la educación a distancia a través de artículos publicados en revistas científicas.

Como ya se señalaba, las Tesis Doctorales proporcionan un buen reflejo de cuál ha podido ser la evolución científica de este campo entre los años 2004-2011. Y los análisis bibliométricos permiten no solo conocer la evolución, sino además analizar con detalle toda la información sobre los temas de estudio que constituyen las actuales líneas de investigación.

\section{RESULTADO Y DISCUSIÓN}

\section{Tesis doctorales defendidas por países y años}

Como se indicaba anteriormente, se ha encontrado un total de 183 tesis doctorales defendidas por doctorados de América Latina sobre el área de la Educación a Distancia. Sin embargo, es importante, antes de analizar en profundidad estos resultados, conocer cómo están distribuidas por años y por países de procedencia de los autores.

Por esta razón en la siguiente tabla se exponen los resultados encontrados analizados por países y por años:

\begin{tabular}{|c|l|l|l|l|l|l|l|l|l|}
\hline & 2004 & 2005 & 2006 & 2007 & 2008 & 2009 & 2010 & 2011 & TOTAL \\
\hline Argentina & 1 & 3 & & & 4 & 1 & 1 & 2 & $\mathbf{1 2}$ \\
\hline Brasil & 23 & 19 & 9 & 9 & 14 & 10 & 14 & 13 & $\mathbf{1 1 1}$ \\
\hline Chile & 2 & 3 & 3 & 1 & 2 & 2 & 1 & 2 & $\mathbf{1 6}$ \\
\hline Colombia & & & & 1 & & & & & $\mathbf{1}$ \\
\hline Cuba & 1 & 2 & 3 & 2 & & 1 & 1 & 2 & $\mathbf{1 2}$ \\
\hline Ecuador & 1 & & & 1 & 1 & & & & $\mathbf{3}$ \\
\hline Honduras & & & & 1 & & & & & $\mathbf{1}$ \\
\hline México & & 3 & $\mathbf{1}$ & $\mathbf{1}$ & $\mathbf{1}$ & $\mathbf{2}$ & $\mathbf{2}$ & $\mathbf{2}$ & $\mathbf{1 2}$ \\
\hline Panamá & & & $\mathbf{1}$ & & & & & & $\mathbf{1}$ \\
\hline Perú & & 2 & & & & & & 1 & $\mathbf{3}$ \\
\hline Venezuela & & 6 & 3 & 2 & 3 & 2 & 1 & $\mathbf{2}$ & $\mathbf{1 9}$ \\
\hline TOTAL & $\mathbf{2 7}$ & $\mathbf{3 5}$ & $\mathbf{2 0}$ & $\mathbf{1 8}$ & $\mathbf{2 1}$ & $\mathbf{1 8}$ & $\mathbf{2 0}$ & $\mathbf{2 4}$ & $\mathbf{1 8 3}$ \\
\hline
\end{tabular}

Tabla 1. Distribución por países de procedencia y años 
En la anterior tabla podemos comprobar que no hay una distribución similar, ni en evolución ni en resultados finales. Si nos centramos en la evolución de los años se observa como existen países donde sí se ha seguido una evolución, más o menos constante en el tiempo de tesis doctorales defendidas en el campo de la educación a distancia. Aunque no exista un alto número de tesis defendidas en algunos países, como por ejemplo en México o Chile, sí que se han defendido trabajos en este campo. Pero sin duda, el país con más alto índice de tesis doctorales defendidas en el campo de la educación a distancia ha sido sin duda Brasil, que además se ha mantenido más o menos constante en el tiempo.

En la siguiente gráfica se observa con mayor detalle la continuidad en el tiempo en estas regiones antes nombradas:

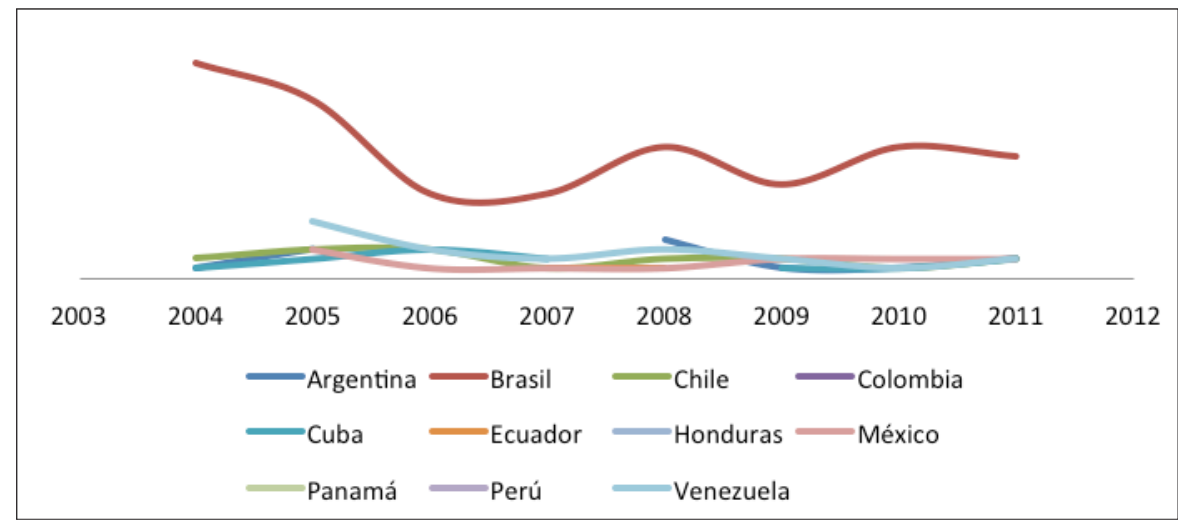

Gráfico 1. Evolución temporal de las tesis doctorales defendidas

Como se mencionaba, Brasil ha sido el país donde se concentran la mayoría de las tesis doctorales, sin embargo, aunque es constante, en los últimos años se puede observar como la producción de este tipo de investigaciones ha decaído. En el caso opuesto se encuentra México, que contando con un menor número, sí ha experimentado una progresión en este campo.

Si nos centramos en los años, momento en que se han defendido las tesis doctorales, encontramos que se ha mantenido constante, aunque se puede decir que no ha existido un incremento si comparamos los años. En 2004 se defendieron 27 tesis doctorales, mientras que en el último año estudiado, 2011, el número descendió (24 tesis doctorales defendidas), aunque no significativamente. 
Un año que es relevante es 2005 momento en que fueron defendidas 35 tesis doctorales, casi el doble que en el año 2007 y 2009. Desconocemos las razones por las que se ha producido en este año un incremento tan significativo.

No obstante, hay que destacar que no existe una tendencia ni positiva ni negativa en términos de progresión lineal, ya que no es significativo el cambio en el número de tesis doctorales defendidas en los años analizados. El número de tesis doctorales defendidas se mantiene, aunque en los últimos años, teniendo un pequeño aumento en los años 2010 y 2011 respecto al año 2008 y 2009, pero en un nivel bajo ( $\rho$ de Spearman = 0,69, p <0,05). Es decir, la correlación entre las dos variables temporales es menos de 1 si comparamos los resultados alcanzados entre los años 2010 y 2011 (que son 44) frente a los años 2008 y 2009 (con un total de 39 tesis doctorales defendidas).

Y finalmente dentro de este apartado debemos analizar los datos en función de los porcentajes. Se presenta a continuación la siguiente tabla donde se ven los resultados finales de las tesis doctorales leídas en función del país de procedencia del autor.

\begin{tabular}{|c|c|}
\hline & Porcentajes \\
\hline Argentina & $\mathbf{6 , 5 5} \%$ \\
\hline Brasil & $\mathbf{6 0 , 6 5 \%}$ \\
\hline Chile & $\mathbf{8 , 7 4} \%$ \\
\hline Colombia & $\mathbf{0 , 5 5} \%$ \\
\hline Cuba & $\mathbf{6 , 5 5} \%$ \\
\hline Ecuador & $\mathbf{1 , 6 4} \%$ \\
\hline Honduras & $\mathbf{0 , 5 5} \%$ \\
\hline México & $\mathbf{6 , 5 5} \%$ \\
\hline Panamá & $\mathbf{0 , 5 5} \%$ \\
\hline Perú & $\mathbf{1 , 6 4} \%$ \\
\hline Venezuela & $\mathbf{1 0 , 3 8 \%}$ \\
\hline TOTAL & $\mathbf{1 0 0 \%}$ \\
\hline
\end{tabular}

Tabla 2. Resultados en porcentajes de las tesis doctorales defendidas

Como se observa en la tabla y en la gráfica siguiente, la gran mayoría de las tesis doctorales analizadas en esta investigación se concentran en Brasil con un 60,65\% del total. El siguiente país que concentra mayor número de tesis doctorales leídas 
en el campo de la educación a distancia entre los años 2004 a 2011 es Venezuela, aunque es evidente que su porcentaje es mucho menor en comparación con Brasil, ya que solo es de un 10,38\% del total.

El siguiente país que le sucede en porcentajes es Chile, pero tan solo con un $8,78 \%$. Seguidamente encontramos tres países con iguales porcentajes $(6,55 \%)$ que son: Cuba, México y Argentina. En el siguiente gráfico se observan las diferencias de porcentajes de forma más clara.

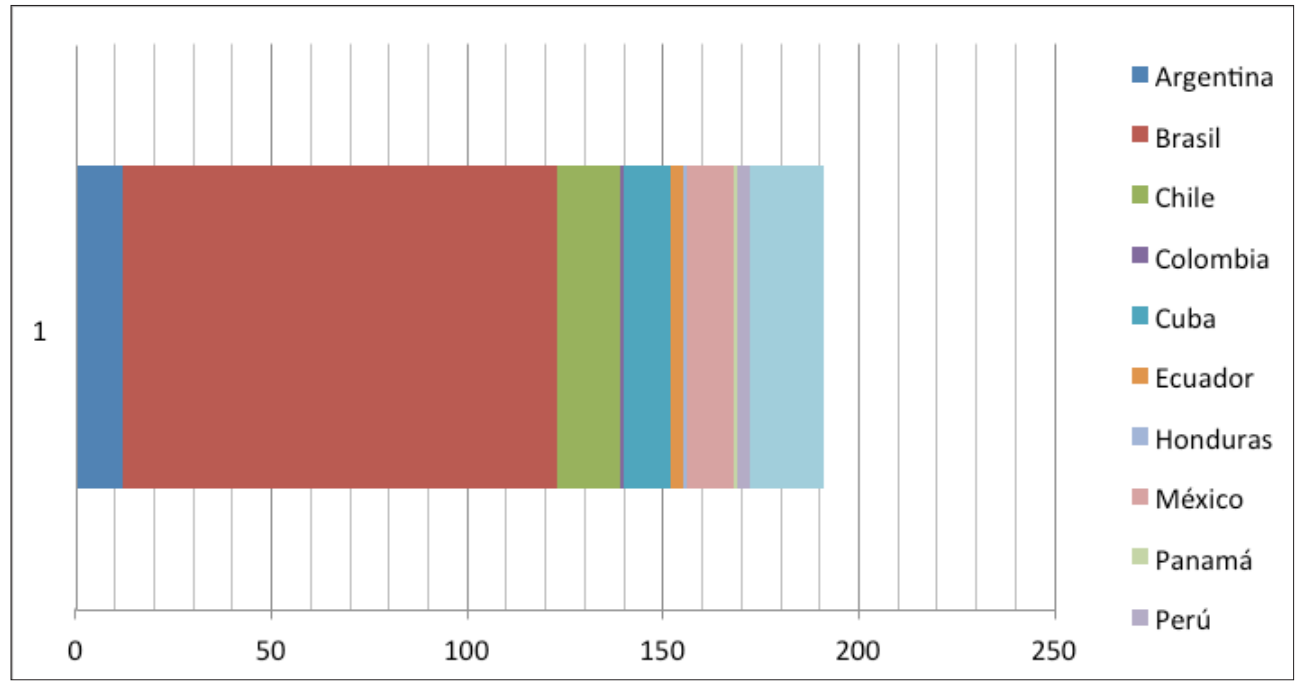

Gráfico 2. Distribución del total de tesis doctorales defendidas por países de América Latina sobre la temática de la Educación a Distancia

Es lógico pensar, y así los hechos lo constatan que el idioma mayoritario haya sido el portugués con un 60,65\% del total, frente a un 39,35\% de las tesis doctorales que han sido publicadas y defendidas en español. Estos datos, vienen producidos por el sesgo diferencial de los documentos analizados, donde ampliamente eran casi todos de origen brasileño. Sin embargo, aunque este hecho no es de gran relevancia en esta investigación, en otras investigaciones llevadas a cabo, como Nascimiento (2000), se detectaron que en las tesis doctorales sobre Ciencias Sociales y Jurídicas, donde estaría enmarcada la educación a distancia, existe un predominio del idioma español.

A continuación se presenta una tabla con el nombre de las universidades donde se han defendido las tesis doctorales. 


\begin{tabular}{|ll|}
\hline \multicolumn{1}{|c|}{} & \multicolumn{1}{|c|}{ Universidades donde se han defendido las tesis doctorales } \\
\hline 1. & Benemérita Universidad Autónoma de Puebla \\
\hline 2. & Pontificia Universidad Católica del Ecuador \\
\hline 3. & Pontifícia Universidade Católica Do Rio Grande Do Sul \\
\hline 4. & Universidad Autónoma de Barcelona \\
\hline 5. & Universidad Barcelona \\
\hline 6. & Universidad Burgos \\
\hline 7. & Universidad Católica de Rio Grande do Sul \\
\hline 8. & Universidad Católica Don Bosco \\
\hline 9. & Universidad Central “Marta Abreu” De Las Villas \\
\hline 10. & Universidad Central de Venezuela \\
\hline 11. & Universidad De Buenos Aires \\
\hline 12. & Universidad De Chile \\
\hline 13. & Universidad de Las Tunas \\
\hline 14. & Universidade Federal do Rio Grande do Sul \\
\hline 15. & Universidad De San Carlos \\
\hline 16. & Universidad Del Estado De Rio De Janeiro \\
\hline 17. & Universidad Estatal de Campinas \\
\hline 18. & Universidad Federal de Bahía \\
\hline 19. & Universidad Federal de Pernambuco \\
\hline 20. & Universidad Federal de Santa Catarina \\
\hline 21. & Universidad Granada \\
\hline 22. & Universidad Málaga \\
\hline 23. & Universidad Metodista de Sau Paulo \\
\hline 24. & Universidad Nacional de La Plata \\
\hline 25. & Universidad Nacional Educación a Distancia \\
\hline 26. & Universidad Popular Autónoma del Estado de Puebla \\
\hline 27. & Universidad Rovira i Virgili \\
\hline 28. & Universidad Salamanca \\
\hline 29. & Universidad Santiago de Compostela \\
\hline 30. & Universidade Sau Paulo \\
\hline 31. & Universidad Sevilla \\
\hline 32. & Universidade Tuiuti Do Paraná \\
\hline & Thbla 3 Universidades donde se han defendido las tesis dotorales \\
\hline
\end{tabular}

Tabla 3. Universidades donde se han defendido las tesis doctorales 
Las tesis producidas se han defendido en 32 universidades diferentes (véase en la tabla anterior), por esta razón, estamos ante un análisis científico de amplia difusión y podemos ver cómo el área de la educación a distancia es estudiada en muchas universidades de América Latina y de España. No obstante un número reducido de universidades concentran el 68\% del total, como es el caso de la Universidade Federal do Rio Grande do Sul y la Universidade Sau Paulo, y en muchas son casos aislados, como en el caso de la Universidade Tuiuti Do Paraná, Pontificia Universidad Católica del Ecuador, Universidad de Las Tunas, o la Universidad Central "Marta Abreu” de Las Villas.

Hubiera sido interesante analizar con detalle los directores de las tesis doctorales y los tribunales de composición de estos para descubrir con más detalle el análisis de redes sociales existentes, sin embargo, esto no se ha podido llevar a cabo a causa de la dispersión encontrada en estos equipos al encontrarse multitud de casos aislados. Esta es una parte que queda pendiente para futuras investigaciones donde se pueda analizar a largo plazo y contar con más tesis doctorales concentradas en regiones más específicas.

\section{Análisis temático}

A partir del estudio elaborado por Zawacki-Richter (2009) se establecieron las categorías para realizar el análisis temático y así conocer las tendencias, prospectiva y necesidades de la educación a distancia. Este sistema de clasificación elaborado por este investigador está basado en otras investigaciones realizadas y que han sido utilizadas en este tipo de estudios. Zawacki-Richter (2009) utilizó los estudios realizados y las clasificaciones elaboradas por Lee, Driscoll y Nelson (2004), Jerez (1995), Phipps y Merisotis (1999) y Khan (1997). A partir de las categorías comunes encontradas elaboró esta clasificación mediante la consulta a expertos a través del método Delphy. En la actualidad, esta clasificación es una de las más utilizadas por encontrarse actualizada y ser la más completa para la situación de la educación a distancia.

Zawacki-Richter (2009) se basó en 15 categorías distribuidas en tres dimensiones, que a continuación se explicará cada una con detalle.

- NiVEL MACRO. LOS SISTEMAS DE EDUCACión A DiSTANCIA Y SUS TEORÍAS

- Acceso, equidad y ética: son los temas relacionados con la democratización de acceso a la educación, infraestructuras insuficientes en 
zonas en desarrollo, brecha digital en términos de acceso a la información, políticas educativas, etc.

- Aspectos relacionados con la globalización y la educación intercultural dentro de la educación a distancia (se refiere al desarrollo del mercado mundial y sus implicaciones para el desarrollo profesional).

- Sistemas institucionales de educación a distancia (son los temas relacionados con la internacionalización, los programas transnacionales, alianzas institucionales y de países en torno a la educación a distancia, redes de colaboración institucional, programas pertenecientes a varias universidades con modalidad a distancia).

- Teorías y modelos: marcos teóricos para las fundamentaciones de la educación a distancia.

- Investigación en educación a distancia y transferencia de conocimiento: consideraciones metodológicas, el impacto de la investigación en la educación a distancia, revisiones de la literatura científica en este ámbito, historia de la educación a distancia y el papel de las asociaciones profesionales con sus informes de investigación.

- $\quad$ NIVEL MEDIO. Gestión, organización y tecnología

- Gestión y organización: organización, planificación y administración de las infraestructuras para la implementación de programas de educación a distancia. Todo lo referente a las políticas institucionales y normativas internas.

- Costes y beneficios: Gestión financiera, becas de matrícula, becas de adquisición de material, modelos de costes, de negocio y de rentabilidad en los programas de educación a distancia.

- Nuevas tendencias de la tecnología dentro de la educación a distancia: nuevas herramientas, selección de recursos, beneficios y retos de utilización, infraestructura técnica y equipos, etc.

- Innovación y cambio: innovación educativa con nuevos medios y medidas, promoción de investigaciones, promoción de cambios, etc. 
- Profesores: formación de profesores, competencias necesarias, desarrollo profesional como requisito previo a la innovación, resistencias al cambio, tutores/formadores, equipos multidisciplinares, funciones, etc.

- Servicios de apoyo al estudiante: asesoramiento al estudiante, medidas de apoyo, bibliotecas, servicio técnico de salidas laborales, asociaciones de alumnos, etc.

- Calidad: cuestiones relativas a los estándares de acreditación y calidad de la educación a distancia. Reputación y credibilidad de la educación a distancia como medio de medir la calidad.

- NIVEL MICRO. Enseñanza y aprendizaje en la educación a distancia

- Diseño instruccional (diseño en el plan de estudios y en el desarrollo del curso, enfoques pedagógicos, diseño de los materiales de estudio, aplicaciones tecnológicas para la enseñanza y el aprendizaje, etc.).

- Interacción y comunicación en las comunidades de aprendizaje: temas relacionados con la interacción, comunicación, relaciones entre alumnos-alumnos y profesores/tutores-alumnos. Y otros aspectos interculturales de la comunicación en línea.

- Características del alumno: estilos de aprendizaje, competencias necesarias, rasgos demográficos, rasgos de personalidad, necesidades especiales, dificultades de aprendizaje, etc.

A partir de estas categorías y subcategorías se fueron clasificando cada una de las tesis doctorales analizadas. Para ello se utilizó Microsoft Excel dividiéndolo por años nuevamente para encontrar las tendencias y conocer aquellas áreas dentro de la educación a distancia que aún no han sido investigadas a través de las tesis doctorales en el campo de la educación a distancia por los doctorandos de América Latina. Se presenta a continuación la tabla correspondiente dividida por años, categorías y subcategorías, así como el número y porcentaje. 


\begin{tabular}{|c|c|c|c|c|c|c|c|c|c|c|c|}
\hline Categoría & Subcategoría & 2004 & 2005 & 2006 & 2007 & 2008 & 2009 & 2010 & 2011 & $\mathrm{~N}$ & $\%$ \\
\hline \multirow{5}{*}{ 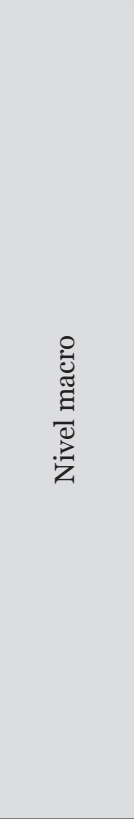 } & $\begin{array}{l}\text { Acceso, equidad } \\
\text { y ética }\end{array}$ & & 1 & & & & & & o & 1 & 0,54 \\
\hline & $\begin{array}{l}\text { Aspectos } \\
\text { relacionados } \\
\text { con la } \\
\text { globalización } \\
\text { y la educación } \\
\text { intercultural } \\
\text { dentro de la } \\
\text { educación a } \\
\text { distancia }\end{array}$ & 1 & 1 & 1 & & 1 & 1 & 1 & 1 & 7 & 3,82 \\
\hline & $\begin{array}{l}\text { Sistemas } \\
\text { institucionales } \\
\text { de educación a } \\
\text { distancia }\end{array}$ & 1 & 4 & 1 & 1 & 1 & & 1 & o & 9 & 4,91 \\
\hline & $\begin{array}{l}\text { Teorías y } \\
\text { modelos }\end{array}$ & & 2 & 2 & & & & & & 4 & 2,18 \\
\hline & $\begin{array}{l}\text { Investigación } \\
\text { en educación } \\
\text { a distancia y } \\
\text { transferencia de } \\
\text { conocimiento }\end{array}$ & & & & & & & & & & \\
\hline \multirow{9}{*}{ 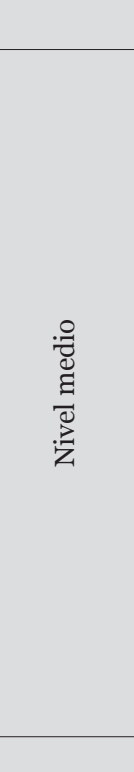 } & & & & & & & & & & 21 & 11,49 \\
\hline & $\begin{array}{l}\text { Gestión y } \\
\text { organización }\end{array}$ & & 2 & & 1 & 1 & & & & 4 & 2,18 \\
\hline & $\begin{array}{l}\text { Costes y } \\
\text { beneficios }\end{array}$ & 1 & 1 & & & & & & & 2 & 1,09 \\
\hline & $\begin{array}{l}\text { Nuevas } \\
\text { tendencias de } \\
\text { la tecnología } \\
\text { dentro de la } \\
\text { educación a } \\
\text { distancia }\end{array}$ & 7 & 6 & 3 & 5 & 5 & 5 & 4 & 7 & 42 & 22,95 \\
\hline & $\begin{array}{l}\text { Innovación y } \\
\text { cambio }\end{array}$ & 2 & 2 & & & & & & & 4 & 2,18 \\
\hline & Profesores & 4 & 4 & 3 & 2 & 4 & 3 & 4 & 4 & 28 & 15,3 \\
\hline & $\begin{array}{l}\text { Servicios } \\
\text { de apoyo al } \\
\text { estudiante }\end{array}$ & 1 & & 1 & 1 & & 1 & 1 & 2 & 7 & 3,82 \\
\hline & Calidad & 1 & 2 & 1 & 1 & 1 & 1 & 1 & 2 & 10 & 5,46 \\
\hline & & & & & & & & & & 97 & 53 \\
\hline
\end{tabular}




\begin{tabular}{|c|l|l|l|l|l|l|l|l|l|l|c|}
\hline Categoría & Subcategoría & $\mathbf{2 0 0 4}$ & $\mathbf{2 0 0 5}$ & $\mathbf{2 0 0 6}$ & $\mathbf{2 0 0 7}$ & $\mathbf{2 0 0 8}$ & $\mathbf{2 0 0 9}$ & $\mathbf{2 0 1 0}$ & 2011 & $\mathrm{~N}$ & $\%$ \\
\hline & $\begin{array}{l}\text { Diseño } \\
\text { instruccional }\end{array}$ & 1 & 1 & 1 & & & & & & 3 & 1,63 \\
\cline { 2 - 14 } & $\begin{array}{l}\text { Interacción y } \\
\text { comunicación } \\
\text { en las } \\
\text { comunidades } \\
\text { de aprendizaje }\end{array}$ & 4 & 5 & 4 & 5 & 6 & 4 & 5 & 5 & 38 & 20,76 \\
\cline { 2 - 12 } & $\begin{array}{l}\text { Características } \\
\text { del alumno }\end{array}$ & 4 & 4 & 3 & 2 & 2 & 3 & 3 & 3 & 24 & 13,11 \\
\hline & & $\mathbf{2 7}$ & $\mathbf{3 5}$ & $\mathbf{2 0}$ & $\mathbf{1 8}$ & $\mathbf{2 1}$ & $\mathbf{1 8}$ & $\mathbf{2 0}$ & $\mathbf{2 4}$ & $\mathbf{1 8 3}$ & $\mathbf{1 0 0}$ \\
\hline
\end{tabular}

Tabla 4. Análisis de las tesis doctorales según la clasificación de Zawacki-Richter (2009)

La tabla anterior revela que no existe un equilibrio entre las 3 categorías. A través de las tesis doctorales se descubre que se ha estudiado en profundidad especialmente el nivel medio, donde más del 50\% (exactamente el 53\%) de las tesis doctorales se han centrado en esta categoría. Seguidamente, a través de las tesis doctorales se ha investigado el nivel macro con un resultado del $35,51 \%$ respecto del total, lo que hace que tan solo se hayan centrado en la investigación a nivel micro un $11,49 \%$. Estos resultados son comparables con otras investigaciones realizadas por ZawackiRichter (2009) o por Gross (2011) donde los resultados alcanzados revelan un fuerte desequilibrio.

Si analizamos las subcategorías también encontramos nuevamente diferencias significativas. Las dos subcategorías que han tenido mayores porcentajes han sido "Nuevas tendencias de la tecnología dentro de la educación a distancia" con un 22,95\% y la subcategoría "Interacción y comunicación en las comunidades de aprendizaje" con un 20,76\%. Este porcentaje coincide parcialmente con la investigación de Zawacki-Richter (2009) que analizaba artículos de revistas científicas, pero que también encontró que las investigaciones se centraban en la subcategoría "Interacción y comunicación en las comunidades de aprendizaje". Y finalmente las últimas subcategorías que destacan frente a las demás son aquellas investigaciones que se han realizado para conocer y analizar con detalle las necesidades y características de los discentes y los docentes dentro de la educación a distancia, que cuenta con unos porcentajes del 13,11 y el 15,3 respectivamente.

Sin embargo, otras sub-categorías se han mantenido en el tiempo, y aunque se sigue investigando sobre ellas no han tenido un incremento significativo. Por ejemplo, el tema de la calidad (con un 5,46 \%) y los servicios de apoyo al estudiante 
(con un 3, 82\%), ambos conceptos muy relacionados entre sí. Y otra subcategoría que está en la misma situación es "Aspectos relacionados con la globalización y la educación intercultural dentro de la educación a distancia” que hay investigaciones sobre este sub-área pero de forma muy limitada.

Es relevante también observar que otras sub-categorías están desapareciendo o no se han encontrado tesis doctorales sobre estas áreas. Desde el año 2004 hasta el año 2011 se han producido escasas investigaciones en las subcategorías: Diseño instruccional (con un 1,63\%), Gestión y organización (2,18 \%), y Costes y beneficios (1,09 \%). Y las investigaciones realizadas en estas sub-categorías se producen en los primeros años analizados (2004, 2005 y 2006).

Y para concluir este apartado es necesario destacar que existen dos subcategorías donde no se ha encontrado ninguna tesis doctoral sobre estos temas. La "Investigación en educación a distancia y transferencia de conocimiento" y el "Acceso, equidad y ética" son dos de las áreas que no han sido investigadas a través de las tesis doctorales analizadas. Estos temas se relación con la democratización de acceso a la educación, infraestructuras insuficientes en zonas en desarrollo, brecha digital en términos de acceso a la información, políticas educativas, pero también la producción de la ciencia en el campo de la educación a distancia y la transferencia de los conocimientos.

\section{El análisis de referencias bibliográficas}

El objetivo de esta parte del trabajo es analizar las referencias bibliográficas incluidas en las tesis doctorales defendidas entre los años 2004-2011 por doctorandos de América Latina en el campo de la educación a distancia. Antes de comenzar su análisis es fundamental distinguir las diferencias entre la referencia bibliográfica y la cita, y para ello se utiliza el concreto que aportó Smith (1981: 83) cuando señala que "una referencia es el reconocimiento que un documento le da a otro y una cita es el reconocimiento que un documento recibe desde otro".

La idea de contar citas que se producen dentro de un mismo texto para evaluar el uso de la literatura no es nueva, ya que encontramos un artículo usando esta técnica, publicado por Gross y Gross en 1927. Según Koenig (1978) "las citas recibidas por artículos anteriores pueden ser usadas para predecir el uso en el presente o en el futuro de los artículos" y por tanto, vienen a ser un reconocimiento indirecto de su relevancia. Por eso consideramos de especial interés realizar el análisis de referencias bibliográficas para poder obtener unos resultados contrastados. 
Los indicadores que utilizamos en el análisis de referencias bibliográficas fueron:

- Número de referencias

- Tipología documental

\section{Número de referencias}

Las tesis doctorales muestran el uso de información y consumo de la ciencia, por ello el número de referencias bibliográficas son un indicador idóneo para conocer los documentos que son utilizados en la actualidad. Las tesis doctorales destacan precisamente por ser investigaciones que utilizan una gran cantidad de referencias bibliográficas muy actualizadas y adecuadamente citadas.

El indicador utilizado para medir la tasa de referencias por publicación es sencillo, aunque su elaboración es costosa. Se calcula dividiendo el número total de referencias entre el número total de documentos fuente. Según Lopez Piñeiro (1992) este indicador es un indicador de producción de la investigación, aunque también puede ser utilizado como indicador de consumo.

Para la elaboración del número total de referencias se realizó a través de una hoja de calcula de Microsoft Excell 2007 donde se recogía la cita completa de cada uno de las fuentes documentales utilizadas, que en este caso son las propias tesis doctorales. Una vez que se obtuvo el resultado se ordenaron las referencias para que no existieran erratas en las propias citas y para eliminar duplicados.

Como resultado final, de las 183 tesis doctorales analizadas, el total de referencias citadas fue de 34.184 , lo que hace una media de 186,8 referencias citadas por cada uno de los documentos utilizados como fuentes primarias.

\section{Tipología documental: tipo de documento citado}

Para conocer el consumo de información por parte de los investigadores en el campo de la educación a distancia, resulta muy interesante utilizar este indicador. Dentro de este indicador es necesario conocer la distribución porcentual de la tipología documental citada, y para ello, es necesario el recuento de las frecuencias obtenidas en el análisis de las referencias bibliográficas y se expresa finalmente de forma porcentual. 
Por tanto, en cuanto a los tipos de documentos citados, si analizamos estos datos, que se pueden ver en la tabla 5 y el gráfico 3, encontramos que el mayor número de citas se centra en los libros (con un 41,83\%), seguido muy de cerca por los artículos publicados en revistas científicas que acumula el 38,93\%. Sin embargo, como se ve reflejado en la tabla, en las tesis doctorales defendidas sobre el ámbito de la educación a distancia por doctorandos de América Latina, existen escasas referencias a otras tesis doctorales que es el valor que menor porcentaje acumula, con tan solo el o,31.

\begin{tabular}{|l|l|l|}
\hline \multicolumn{1}{|c|}{ Tipo de citación } & \multicolumn{1}{c|}{ Número de citas } & \multicolumn{1}{c|}{ Porcentaje } \\
\hline Artículos de revistas & 12.528 & $38,93 \%$ \\
\hline Capítulos de libros & 4.033 & $12,53 \%$ \\
\hline Libros & 13.461 & $41,83 \%$ \\
\hline Actas de congresos & 1.730 & $5,37 \%$ \\
\hline Tesis doctorales & 102 & $0,31 \%$ \\
\hline Otros & 2.051 & $6,37 \%$ \\
\hline Total & $\mathbf{3 2 . 1 7 5}$ & $\mathbf{1 0 0} \%$ \\
\hline
\end{tabular}

Tabla 5. Tipos de documentos citados

Otros valores que han tenido escasas referencias son lo que se ha llamado Actas de congresos (con un 5,37\%) donde se encuentran actas de reuniones, ponencias y presentaciones de congresos, workshop y seminarios. En el apartado Otros encontramos recursos Web, software, informes de organismos internacionales y nacionales, y otros documentos electrónicos que se encontrarían dentro de la literatura gris. Este apartado otros tiene también un escaso porcentaje en las referencias de citas, alcanzando el 6,37\%.

En la siguiente gráfica 3 se puede ver la distribución del análisis de referencias encontradas en las tesis doctorales. 


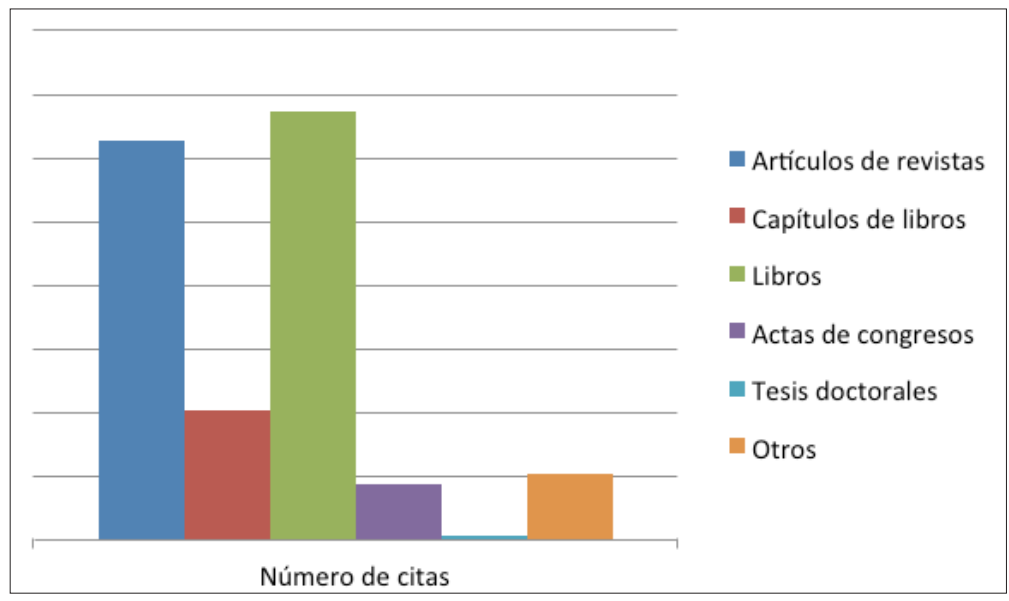

Gráfica 3. Tipo de documento referenciado en las citas

En otras investigaciones que se basan en el análisis de citas en las tesis doctorales se encontraron resultados similares, aunque no idénticos. En la gran mayoría de las investigaciones, los artículos publicados en las revistas científicas y los libros tienen un alto índice de referencias en las citas. Por ejemplo, Haycock (2004) y Vallejo (2006) también observaron en sus estudios que las fuentes bibliográficas utilizadas mayoritariamente fueron los libros. Sin embargo, en el trabajo de Feyereisen (2009) al igual que en este, se detectó que los libros tienen un alto porcentaje frente a otras fuentes de información como pueden ser las referencias a otras tesis doctorales publicadas en la misma área.

El análisis de referencias del llamado apartado Otros también ha sido analizado en diversas investigaciones, como en el caso de Gao y otros (2009) y Klassen (2011). Ambos autores llegan a la misma conclusión, y es el sorprendente aumento de citas a este tipo de recurso. Igualmente consideran que la causa de ese alto porcentaje se debe al incremento en el uso de los recursos electrónicos a través de Internet y el acceso rápido que puede tener un investigador a este tipo de documentos.

\section{CONCLUSIONES}

Hemos estudiado, utilizando como fuente primaria las tesis doctorales defendidas por doctorandos de América Latina entre los años 2004-2011, las áreas de investigación en la educación a distancia, las tendencias, las áreas prioritarias y las lagunas en la investigación de la educación a distancia, pero además se ha realizado un análisis de referencias de citas utilizadas en dichos documentos como fuente de consumo y producto de la investigación en este campo. 
Los resultados alcanzados revelan que no ha existido un aumento significativo en el número de tesis doctorales en el campo de la educación a distancia durante los años estudiados, sino que la tendencia se ha mantenido. No obstante, hay que destacar que de las 183 tesis doctorales analizadas más del 6o\% correspondían a doctorandos de una misma región, y en mucha menor medida se encuentran el resto de los países. Una de las razones por las que no se han analizado las tesis doctorales de otros países de América Latina puede ser a causa de que no se encuentran en bases de datos digitalizadas o en repositorios institucionales, por lo que no se puede concluir que no se defiendan tesis doctorales en el área de la educación a distancia en el resto de los países de esta región, sino que no se ha podido acceder a ellos para analizarlos.

Por otro lado, las tesis doctorales se han defendido en 32 universidades diferentes, aunque se concentraban especialmente en universidades brasileiras. Como pudo observarse muchos doctorandos de América Latina defienden sus tesis doctorales fuera de su país de origen, en concreto en universidades españolas como la Universidad Nacional de Educación a Distancia (UNED) lugar de celebración de gran parte de las defensas de tesis en España.

En investigaciones futuras se propone analizar con detalle los directores de las tesis doctorales y los tribunales de composición de estos para tratar de descubrir el análisis de redes sociales existentes, sin embargo, esto no se ha podido llevar a cabo a causa de la dispersión encontrada en estos equipos. Esta es una parte que queda pendiente para futuras investigaciones donde se pueda analizar a largo plazo y contar con más tesis doctorales concentradas en regiones más específicas.

Respecto a los resultados del análisis temático se detecta una gran concentración de tesis doctorales en áreas muy específicas, y se centran especialmente en los patrones de interacción y comunicación que se producen en esta modalidad educativa, de ahí que también destaque las nuevas herramientas que se crean como parte de la tecnología educativa para establecer nuevos canales de comunicación con los estudiantes. Y finalmente la última subcategoría que destaca frente a las demás es la relativa a aquellas investigaciones que se han realizado para conocer y analizar con detalle las necesidades y características de los discentes dentro de la educación a distancia.

Es relevante también observar que otras sub-categorías están desapareciendo o, al menos, no se han encontrado tesis doctorales sobre estas áreas. Desde el año 2004 hasta el año 2011 se han producido escasas investigaciones en las subcategorías: Diseño instruccional, Gestión y organización, y Costes y beneficios. 
Y las investigaciones realizadas en estas sub-categorías se producen en los primeros años analizados (2004, 2005 y 2006).

Otro aspecto a destacar dentro de las conclusiones es la necesidad de investigar en torno a la "Investigación en educación a distancia y transferencia de conocimiento" y el "Acceso, equidad y ética", ya que los resultados obtenidos manifiestan que son dos de las áreas que no han sido investigadas a través de las tesis doctorales analizadas, y que ahora mismo son muy interesantes para toda la comunidad científica por el gran cambio que se ha producido en los últimos años.

Y finalmente hay que destacar el análisis de referencias bibliográficas como fuente de consumo de información de los investigadores en el campo de la educación a distancia. Se detectó que el mayor número de citas se centra en los libros, seguido muy de cerca por los artículos publicados en revistas científicas, sin embargo, existen escasas referencias a otras tesis doctorales. Estos resultados concuerdan con otras investigaciones realizadas en el campo de las ciencias sociales.

\section{REFERENCIAS BIBLIOGRÁFICAS}

Anglin, G. J.; Morrison, G. (2000). Un análisis de la investigación en educación a distancia: Implicaciones para el tecnólogo de instrucción. Revisión Trimestral de Educación a Distancia 1, (189-194).

Berge, Z. L.; Mrozowski, S. (2001). Revisión de la investigación en la educación a distancia, 1990 a 1999. American Journal of Distance Education, 15 (3), (5-19).

Bonk, C. J.; Dennen, V. (2003). Frameworks for research, design, benchmarks, training, and pedagogy in Web-based distance education. In: Moore, M. G.; Anderson, B. (Ed.). Handbook of distance education. Mahwah, NJ: Lawrence Erlbaum Associates.

Buela Casal, G. (2005). Situación actual de la productividad científica de las universidades españolas. International journal of clinical and health psychology, 5 (1), (175-190).

Clark, M. L. (2005). El ISI reconoce el impacto de nuestra revista. Rev Panam Salud Pública, 17(2), (73-74).
Cordón García, J. A. (2009). La edición universitaria en el contexto de la edición científica. En: García Caro, C.; Vilches Pardo, J. (Coord.) Homenaje a Isabel de Torres: estudios de documentación dedicados a su memoria. Granada: Editorial Universidad de Granada, (115-130).

Fernández Quijada, D. (2008). Revistas científicas e índices de impacto. A propósito de «Hacer saber». Área abierta, 20, (1-10).

Feyereisen, P; Spoiden, A. (2009). Can Local Citation Analysis of Master's and Doctoral Theses Help Decision-Making about the Management of the Collection of Periodicals? A Case Study in Psychology and Education Sciences. Journal of Academic Librarianship.

Gao, S. J.; Yu, W. Z.; Luo, F. P. (2009). Citation analysis of $\mathrm{PhD}$ thesis at Wuhan University, China. Library Collections, Acquisitions, and Technical Services, 33 (1), (8-16). 
García Aretio, L. (2014). Bases, mediaciones y futuro de la educación a distancia en la sociedad digital. Madrid: Síntesis.

Gross, P. L. K.; Gross, E. M. (1927). College libraries and chemical education. Science, 661, (385-389).

Haycock, L. A. (2004). Citation analysis of educational dissertations for collection development. Library Resources and Technical Services, 48 (2), (102-106).

Hew, K. F.; Kale, U.; Kim, N. (2007). Past research in instructional technology: Results of a content analysis of empirical studies published in three prominent instructional technology journals from the year 2000 through 2004. Journal of Educational Computing Research, 36 (3), (269-300).

Hranstinski, S.; Keller, C. (2007). An examination of research approaches that underlie research on educational technology: A review from 2000 to 2004. Journal of Educational Computing Research, 36 (2), (175-190).

Jerez, L. (1996). Problemas en el aprendizaje a distancia. Revista Internacional de Telecomunicaciones para la Educación 1 (4), (337-365).

Klassen, T. W. (2011). A citation study of public health Masters' theses. Collection Building, 30 (4), (153-159). [en línea] Disponible en: http://www.emeraldinsight.com/0160-4953.htm [consultado 2013, 09 de septiembre].

Koble, M.A.; Bunker, J. (1997). Tendencias en la investigación y la práctica: un examen de la revista americana de la educación a distancia desde 1987 hasta 1995 . American Journal of Distance Education 11 (2), (19-38).

Koenig, E. D. (1978). Citation analysis for the Arts and Humanities as a collection management tool. Collection Management, 2 (3), (247-261).

Latchem, C. (2006). Editorial: A content analysis of the British Journal of Educational Technology. British Journal of
Educational Technology, 37 (4), (503511).

Lee, Y.; Driscoll, M. P.; Nelson, D. W. (2004). Pasado, Presente y Futuro de la Investigación en Educación a Distancia: Resultados de un Análisis de Contenido. American Journal of Distance Education, 18 (4) (225-241). [en línea] Disponible en: http://www.tandfonline.com/doi/ abs/10.1207/s15389286ajde1804 4 [consultado 2013, o9 de septiembre].

López López, P. (1996). La investigación bibliométrica en España (tesis doctorales). Revista Española de Documentación Científica, 19 (1), (84-89).

López Piñero, J. M.; Terrada, M. L. (1992a). Los Indicadores bibliométricos y la evaluación de la Actividad médico-Científica. (III) Los Indicadores de Producción, Circulación dispersión y, consumo de la Información y Repercusión. Medicina Clínica, 98, (142-148).

López-Piñero, J. M.; Terrada, M. L. (1992b). Los Indicadores bibliométricos y la evaluación de la Actividad médico-Científica. (I) Usos o Abusos de la bibliometría. Medicina Clínica, 98, (64-68).

López-Piñero, J. M.; Terrada, M. L. (1992c). Los indicadores bibliométricos y la evaluación de la actividad médico científica (II). La comunicación científica en las distintas áreas de la ciencia médica. $\mathrm{Me}$ dicina Clínica, 98 (3), (101-106).

Lu, H.; Wu, C.; Chiu, C. (2009). Tendencias de investigación en e-learning 20052007: un análisis de contenido de los artículos publicados en revistas seleccionadas, [en línea] Disponible en: http://www.ntnu.edu.tw/acad/rep/ r98/k980002-1.pdf [consultado 2013, o9 de septiembre].

Ma, Y. (2000). Research in educational communications and technology at the University of Wisconsin: A study of dissertation completed since the inception of the program. The 22'rd National Conven- 
tion of the Association for Educational Communications and Technology.

Moore, M. G.; Anderson, B. (2003). Handbook of distance education. Mahwah, NJ: Lawrence Erlbaum Associates.

Moore, M. G.; Kearsley, G. (2005). Distance Education A Systems View. Thomson Wadsworth.

Moore, N. (2000). How to do research: The complete guide to designing and managing research projects. London: Library Association. $3^{\text {a }}$ Edición.

Moyano, M.; Delgado, C.; Buela, G. (2006). Análisis de la productividad científica de la Psiquiatría española a través de las tesis doctorales en la base de datos TESEO (1993-2002). Internat J Psychol Ther, 6 (1), (111-20).

Nascimento, M. J. (2000). Producción científica brasileña en España: documentación de las tesis doctorales. Ci. Inf, 29 (1), (3-13).

Perraton, H. (2000). Rethinking the research agenda. The International Review of Research in Open and Distance Learning, 1 (1). [en línea] Disponible en: www.irrodl.org/index.php/irrodl/article/view/5/338 [consultado 2013, o9 de septiembre].

Price, D. J. S. (1963). Litle Science, Big Science. New York: Columbia. Univ. Press.

Price, D. J. S. (1978). Toward a model for Science Indicators. In: Elkana, Y. et al. (Ed.). Towards a metric of science. New York: Wiley

Quintanilla-Montoya, A. L. (2010). La ciencia y su producción de conocimiento en América Latina. Revista Investigación ambiental, Ciencia y política pública, 2 (1), (75-84).

Repiso-Caballero, R.; Torres-Salinas, D.; Delgado López-Cózar; E. (2013). La investigación científica sobre cine en españa a partir de sus tesis doctorales: análisis de redes sociales (1978-2007). ICONO, 14, 11 (2).
Rosado Millan, M. J. y otros (2007). Criterios de clasificación de los medios de difusión de la producción académica y científica universitaria. ACAP. [en línea] Disponible en: http://www.madrid.org/cs/Satellite?blobcol=urldata\& blobheader=application\%2Fpdf\&blobh eadername $1=$ Content-Disposition\&blob headervalue $1=$ filename $\% 3$ DFormato $+d$ efinitivo+del+libro+(TECNISER).pdf\&b lobkey $=$ id\&blobtable $=$ MungoBlobs\&blo bwhere $=1196188616655 \&$ ssbinary $=$ true [consultado 2013, 15 de septiembre].

Ross, S. M.; Morrison, G. R.; Lowther, D.L. (2010). Educational technology research past and present: Balancing rigor and relevance to impact school learning. Contemporary Educational Technology, 1 (1), (17-35).

Rourke, L.; Szabo, M. (2002). Un análisis del contenido de la Revista de Educación a Distancia 1986-2001. Revista de Educación a Distancia, 17 (1), (63-74).

Saavedra Fernández, O.; Sotolongo Aguilar, G.; Guzmán Sánchez, M. V. (2002). Medición de la producción científica en América Latina y el Caribe en el campo agrícola y afines: un estudio bibliométrico. Revista española de Documentación Científica, 25 (2).

Salar, H. C. (2009). Tendencias en Investigación de Educación a Distancia en Turquía. Actas de la $23^{a}$ Asamblea Internacional de Educación Abierta ya Distancia (ICDE) Conferencia Mundial. Maastricht, Países Bajos. [en línea] Disponible en: http://www.ou.nl/Docs/ Campagnes/ICDE2009/Papers/Final paper 348salar.pdf [consultado 2013, 15 de septiembre].

Sierra Bravo, R. (1996). Tesis doctorales y trabajos de investigación científica: metodología general de su elaboración y documentación. Madrid: Paraninfo.

Smith, L. C. (1981). Citation analysis. Library Trends. Summer. 
Solano, E. y otros (2009). La bibliometría: una herramienta eficaz para evaluar la actividad científica postgraduada. $R e-$ vista MediSur, 7 (4) Cienfuegos jul-ago.

Stein, D. S.; Wanstreet, C. E.; Krisch, C. (2011). Investigación de la Educación a Distancia en Educación de Adultos revistas: Un Análisis de Contenido. Midwest Investigación a la Práctica Conference Proceedings. Ed. Glowacki MichelleDudka, (178-183). [en línea] Disponible en: http://68.191.206.6/mwr2p/docs/ SteinWanstreetKrisch.pdf [consultado 2013, 15 de septiembre].

Vallejo Ruiz, M.; Fernández Cano, A.; Torralbo Rodriguez, M. (2006). Patrones de citación en la investigación española en educación matemática. Revista Española de Documentación Científica, 29 (3), (382-297).

Zawacki-Richter, O. (2009). Áreas de Investigación en Educación a Distancia: un estudio Delphi. IROLD Revista Inter- nacional de Investigación en Aprendizaje Abierto y a Distancia, 10 (3). [en línea] Disponible en: http://www.irrodl. org/index.php/irrodl/article/viewArticle/674 [consultado 2013, 15 de septiembre].

Zawacki-Richter, O. y otros (2009). Revisión de Investigación de Educación a Distancia (2000 a 2008): Análisis de las áreas de investigación, métodos y modelos de autoría. IROLD Revista Internacional de Investigación en Aprendizaje Abierto y a Distancia 10 (6), (21-50). [en línea] Disponible en: http://www.irrodl.org/ index.php/irrodl/article/view/741/1433 [consultado 2013, 15 de septiembre].

Ziman, J. (1972). El conocimiento público. México: Fondo de Cultura Económica.

Ziman, J. (1980). La enseñanza y el aprendizaje de las ciencias y de la sociedad. Nueva York: Cambridge University Press.

\section{PERFIL ACADÉMICO Y PROFESIONAL DE LOS AUTORES}

María García Pérez Calabuig. Profesora en el Departamento de Teoría de la Educación y Pedagogía Social por la Facultad de Educación de la Universidad Nacional de Educación a Distancia. Es colaboradora de la Cátedra UNESCO de Educación a Distancia (CUED).

E-mail: mgarcia@edu.uned.es

Lorenzo García Aretio. Catedrático de Teoría de la Educación y Educación a Distancia en la Facultad de Educación de la Universidad Nacional de Educación a Distancia. Es director de la Cátedra UNESCO de Educación a Distancia (CUED).

E-mail: cued@edu.uned.es

\section{DIRECCIÓN POSTAL DE LOS AUTORES}

Facultad de Educación

Universidad Nacional de Educación a Distancia, UNED

Calle Juan del Rosal 14, segunda planta

Ciudad Universitaria, Madrid, España 
Fecha de recepción: 15/09/2013

Fecha de aceptación: 12/12/2013

\section{Como citar este artículo:}

García Pérez, M.; Garcia Aretio, L. (2014). Líneas de investigación y tendencias de la educación a distancia en América Latina a través de las tesis doctorales. RIED. Revista Iberoamericana de Educación a Distancia, volumen 17, $\mathrm{n}^{0}$ 1, pp. 201-230. 